chung [Kronenbuerger et al. Mov Disord 2015; 14: 1125 - 30]. Die Autoren untersuchten 10 Patienten mit ParkinsonSyndrom oder essenziellem Tremor und THS (18 Elektroden) mit bis zu 7,5-jährigem Verlauf post mortem. Dabei fanden sie um den Elektrodenschaft bei al- len Patienten eine Astrogliose, bei $93 \%$ Lymphozyten und bei $68 \%$ multinukleäre Riesenzellen und Makrophagen. Zudem fielen an den Elektrodenspitzen Axonauftreibung und eine erhöhte Expression des Amyloid-Vorläufer-Proteins auf. Patienten, die im Rahmen einer
Sepsis starben, zeigten eine stärkere Astrogliose und mehr nukleäre Riesenzellen, als Patienten, die aus kardiovaskulärer Ursache starben. Ute Ayazpoor

Bewegungsstörungen (Jörg B. Schulz), 8. Neurologie-Update-Seminar, Mainz, 19.-20.2.2016

\title{
Nicht motorische Störungen bei Myasthenia gravis
}

\begin{abstract}
Neben der charakteristischen körperlichen Schwäche und zunehmenden Ermüdbarkeit der Muskulatur können bei Myastheniepatienten auch Riechfunktion, Geschmackssinn und die Schlafqualität beeinträchtigt sein.
\end{abstract}

E ine türkische Arbeitsgruppe prüfte - die Riechfunktion und das Geschmacksvermögen von 30 Patienten mit Myasthenia gravis im Vergleich zu 30 gesunden Freiwilligen mit Riechstiften beziehungsweise Geschmacksstreifen. Die Ergebnisse wurden quantitativ analysiert [Tekeli $\mathrm{H}$ et al. J Neurological Sciences 2015; 356: 188 -92].

Die Untersuchung deckte bei vier Myastheniepatienten eine Anosmie und bei 26 eine Hyposmie auf, unter den gesunden Kontrollen wurde dagegen keine Beeinträchtigung festgestellt. Die Überprüfung des Rauchverhaltens ergab keine Unterschiede zwischen beiden Gruppen. Auch bei der Geschmacksprobe - es mussten vier Qualitäten unterschieden werden - schnitten die Myasthenie-Patienten schlechter ab: Ihre Fähigkeit zur Diskrimination und Identifikation war gegenüber den gesunden Kontrollen signifikant reduziert und ihre Geschmacksschwelle war deutlich angehoben. Über die Genese dieser Riech- und Geschmacksstörung kann laut Professor Heinz Reichmann, Direktor der Klinik und Poliklinik für Neurologie, Universitätsklinikum Carl Gustav Carus, Dresden, bislang nur spekuliert werden. Verantwortlich könnte die insbesondere für den Geschmackssinn wichtige Acetylcholinfunktion sein. Eine andere Möglichkeit wäre, dass AcetylcholinrezeptorAntikörper im Bereich der olfaktorischen Bahnen und der Geschmacksbah- nen eingreifen. Schließlich sei es durchaus denkbar, dass Medikamente, etwa Steroide, eine Dysfunktion der Geruchsund Geschmacksbahnen induzieren, diskutierte Reichmann.

Relevant für die tägliche Praxis sind nach Ansicht von Reichmann auch die Ergebnisse einer weiteren Übersichtsarbeit [Oliveira EF et al. J Phys Ther Sci 2015; 27: 2013 - 8]. Danach schlafen Patienten mit Myasthenia schlechter, sind vermehrt tagesmüde und haben deutlich häufiger Atemstörungen in der Nacht. Er empfahl deshalb, Myasthenie-Patienten zu ihrem Schlaf zu befragen - auch um eine Indikation für eine nächtliche Maskenbeatmung unabhängig von der Muskelschwäche zu erkennen. Ute Ayazpoor

Neuromuskuläre Erkrankungen (Heinz Reichmann), 8. Neurologie-Update-Seminar, Mainz 19. -20.2 .2016$

\section{Nützliche Schmerztherapie bei IPS}

Bis zu $85 \%$ der Patienten mit idiopathischem Parkinson-Syndrom (IPS) klagen über Schmerzen. Besserung verspricht die Behandlung mit Oxycodon/Naloxon, die zum ersten Mal in einer 2015 veröffentlichten Studie geprüft wurde.

chmerzen sind bei Morbus Parkinson nicht nur häufig, sondern präsentieren sich zudem sehr heterogen: Sie können im Rahmen dystoner Muskelkontraktionen in Off-Phasen auftreten, aber auch als muskuläre oder periphere neuropathische und zentrale Schmerzen, berichtete Professor Jörg B. Schulz, Ärztlicher Direktor der Neurologischen Klinik, Universitätsklinikum Aachen.

Schmerzen beeinträchtigen die aufgrund der Grundkrankheit oft schlechte
Lebensqualität der Patienten zusätzlich und sind somit therapiebedürftig, so der Experte für Bewegungsstörungen. Trenkwalder et al. [Lancet Neurol 2015; 14: 1161 - 70] prüften nun in einer ersten klinischen randomisierten placebokontrollierten Phase-II-Studie unter Einschluss von 202 Patienten mit IPS (Hoehn \& Jahr Stadium II-IV) die Therapie mit retardiertem Oxycodon/Naloxon. Die Patienten mussten an mindestens einer Form eines schweren
Schmerzsyndroms leiden und den Schweregrad 6 auf einer Schmerzskala von 0 bis 10 erreichen. Sie erhielten randomisiert (1:) entweder Oxycodon/ Naloxon (von $2 \times 5 \mathrm{mg} / 2,5 \mathrm{mg}$ bis maximal 2 x $20 \mathrm{mg} / 10 \mathrm{mg}$ ) oder Placebo. Beim primären Endpunkt, dem durchschnittlichen 24-Stunden-Schmerzscore nach 16-wöchiger Therapie, schnitt das Verum zwar nicht signifikant besser ab als Placebo: Der durchschnittliche Schmerzscore war auf 5,0 versus 5,6 unter Placebo zurückgegangen $(p=0,058)$. Dieses Ergebnis sei aber durchaus „nützlich für die Praxis“, kommentierte Schulz.

Ute Ayazpoor

Bewegungsstörungen (Jörg B. Schulz), 8. Neurologie-Update-Seminar, Mainz, 19.-20.2.2016 